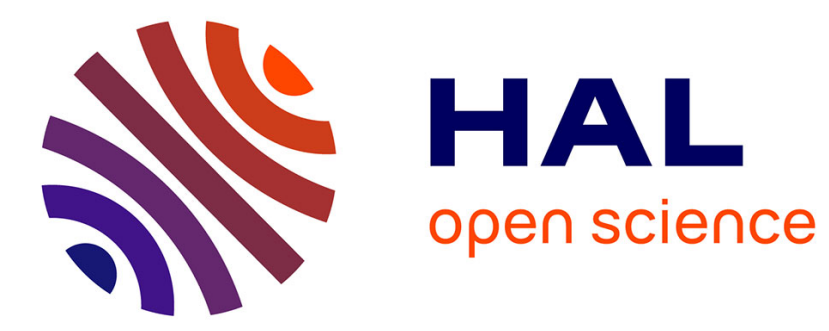

\title{
On detection and representation of resonances by the method of stabilization
}

\author{
A. Macías, A. Riera
}

\section{To cite this version:}

A. Macías, A. Riera. On detection and representation of resonances by the method of stabilization. Journal de Physique, 1985, 46 (4), pp.535-543. 10.1051/jphys:01985004604053500 . jpa-00209993

\section{HAL Id: jpa-00209993 https://hal.science/jpa-00209993}

Submitted on 1 Jan 1985

HAL is a multi-disciplinary open access archive for the deposit and dissemination of scientific research documents, whether they are published or not. The documents may come from teaching and research institutions in France or abroad, or from public or private research centers.
L'archive ouverte pluridisciplinaire HAL, est destinée au dépôt et à la diffusion de documents scientifiques de niveau recherche, publiés ou non, émanant des établissements d'enseignement et de recherche français ou étrangers, des laboratoires publics ou privés. 
Classification

Physics Abstracts

$31.10-31.50$

\title{
On detection and representation of resonances by the method of stabilization
}

\author{
A. Macías $\left({ }^{+}\right)$and A. Riera \\ Depto. Química Física y Química Cuántica/y C.S.I.C. $\left({ }^{+}\right)$ \\ Facultad de Ciencias, Universidad Autónoma de Madrid, Cantoblanco, Madrid, 34, Spain
}

(Reçu le 3 février 1984, révisé le 9 octobre, accepté le 27 novembre 1984)

\begin{abstract}
Résumé. - Nous présentons une explication du succès de la méthode de stabilisation dans la détection et la représentation des résonances. Cette explication est fondée sur le fait qu'une approche variationnelle consiste en une discrétisation à grand pas du continuum qui privilégie des régions particulières de l'espace des configurations. Nous illustrons comment la stabilisation des valeurs propres qui correspondent à des résonances découle simplement des propriétés de la représentation de Heisenberg des fonctions variationnelles. Nous présentons nos conclusions sur les propriétés que les fonctions de base doivent satisfaire afin de pouvoir détecter les résonances et les représenter avec précision.
\end{abstract}

\begin{abstract}
We present an explanation of why the stabilization method works in the detection and representation of resonances. This explanation is based on the fact that a variational approach provides a coarse grain discretization of the continuum which privileges particular regions of configuration space. We illustrate how the stability of eigenvalues corresponding to resonances results simply from the properties of the Heisenberg representation of the variational wavefunctions. Conclusions are drawn as to the properties that basis sets should have to detect resonances and to reproduce them accurately.
\end{abstract}

\section{Introduction.}

In theoretical studies of electron(photon)-atom(molecule) scattering one often has to deal with the existence of resonant states of the composite system. In the same way, the presence of channels which correspond to auto-ionizing molecular states of a given system is not infrequent in the treatment of atom-atom(molecule) collisions. A well known example is provided by Penning ionization, where the excitation energy of one of the partners is greater than the ionization potential of the other; similarly, one often needs to use diabatic states which penetrate into the continuum of the corresponding positive ion. See e.g. the work of O'Malley [1] and a discussion of it in reference [2].

The aim of this paper is to explain the basis of one particular method of calculating resonance energies; the so called stabilization method [3]. As far as we know the heuristic explanation we present is new; and we differentiate between representation of continuum wavefunctions by $L^{2}$ integrable wavefunctions (LIF) and the stability property of certain eigenvalues. To do so, we contrast Fine (FGD) and Coarse (CGD) grain discretizations of the continuum; and we find that stabilization of resonance energies does not require an excellent representation of the continuum wavefunctions (FGD), but a coarser one (CGD) which privileges certain regions of configuration space. Consequences of our treatment concerning the properties of basis sets which are required for a good representation of resonances are drawn.

To make the aims of this paper clear we shall start by presenting two simple instances of stabilization of resonances which allow us to set up the problem, to introduce notation, and to discuss their main common characteristics. These examples allow us to bring up several basic questions as to how the method works. We find the answer to these questions to be based on the properties of the Heisenberg transform of the variational wavefunctions, which in turn is related to the characteristics of both the resonant state and the basis set used in the calculation. Our points are exemplified by variational calculations of the resonance energies in two very simple cases where the exact solutions are known.

Atomic units will be used throughout. 


\section{Setting up the problem.}

We now define what we call the stabilization method. The method consists of varying some non linear parameters in the set of LIF which are used in a variational treatment to describe a resonant state. When this is done, one finds that some eigenvalues of the Hamiltonian matrix are stable (vary little) under these variations and the corresponding wavefunctions are taken as representing resonances.

Let us consider two specific cases which are very simple and yet present different instances of stabilization.

1. Take a (finite) square well potential defined by

$$
\begin{aligned}
& V(r)=-1.125 \text { a.u. } \quad 0 \leqslant r \leqslant 1 \\
& 2.0 \text { a.u. } \quad 1 \leqslant r \leqslant 2 \\
& 0 \quad r>2
\end{aligned}
$$

which has a sharp resonance at $E=1.125$ a.u. Let us diagonalize

$$
H=-\frac{1}{2} \nabla^{2}+V(r)
$$

in an even tempered (that is, with exponents $\alpha_{i}=\alpha_{0} \beta^{i}$, where $\beta$ is a constant) basis [4] of 1s Slater type orbitals (STO).

$$
\varphi_{n, l, m, \alpha_{i}}(\mathbf{r})=N_{i} r^{n-1} \mathrm{e}^{-\alpha_{i} r} Y_{l}^{m}(\theta, \phi)
$$

and we obtain a series of eigenvalues. One then intuits that one of them is related to the position of the resonance, and that it should be stable with respect to variations of a suitable parameter in the basis set; in this case we have chosen an overall scaling parameter $\left({ }^{1}\right)$ of the STO exponents $\left(\alpha_{i}\right.$ in Eq. (3)) which are defined as

$$
\alpha_{i}^{(\eta)}=\alpha_{i} \beta^{\eta} .
$$

This yields different basis sets for different values of $\eta$. All eigenvalues present the same pattern of behaviour; an illustration is given in figure 1 for specific values of $\alpha_{0}, \beta$, and the scaling parameter $\eta$. There is a clear plateau region where the eigenvalue is stable and it does correspond very closely to the resonant energy.

2. Consider the united atom $R=0$ (u.a.) limit $\mathrm{B}^{3+}$ of the quasi-molecule $(\mathrm{LiHe})^{3+}$ currently under study in our group [5], and diagonalize the corresponding Hamiltonian in an even tempered basis set of 1s and 2s STO's. We vary their exponents as in part 1 and find that some eigenvalues which are above the ionization limit corresponding to the energy of the ground state of the monoelectronic ion $\mathrm{B}^{4+}$ remain stable in the neighbourhood of the u.a. limit ${ }^{1} \mathrm{~S}\left(n \mathrm{~s} n^{\prime} \mathrm{s}\right)$ resonant states. We show in figure 2 the variation of one of those eigenvalues.

( ${ }^{1}$ This is just one of the ways of varying the basis set which can be used in stabilization techniques; we are not advocating its use against alternative procedures.

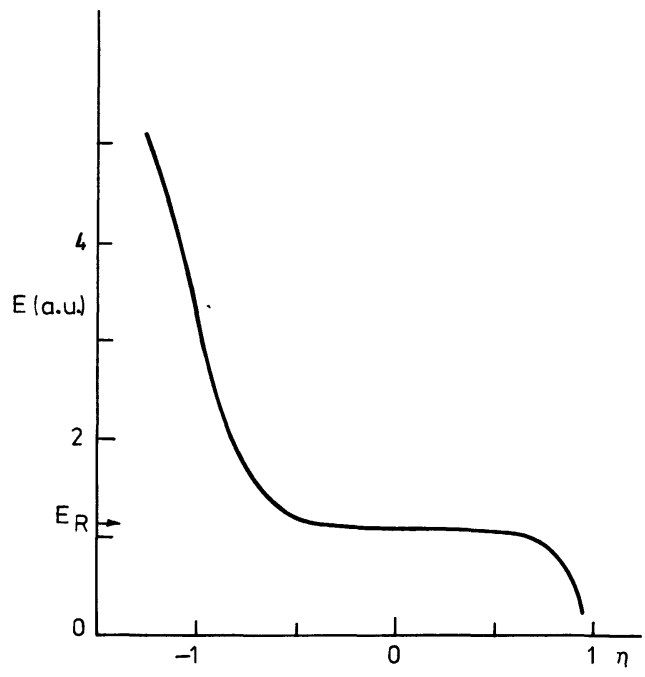

Fig. 1. - Stabilization of one eigenvalue, $E_{7}$, of the Hamiltonian equation (2) with the potential of equation (1). We plot the eigenvalue $v s$. the scaling parameter $\eta$ defined in equation (3). The energy $\left(E_{\mathrm{R}}\right)$ of the sharp resonance of (1) is indicated. Basis set : 1s STO's with $\alpha_{0}=2 ; \beta=2$; $i=0, \cdots+5$ (see Eq. (3)).

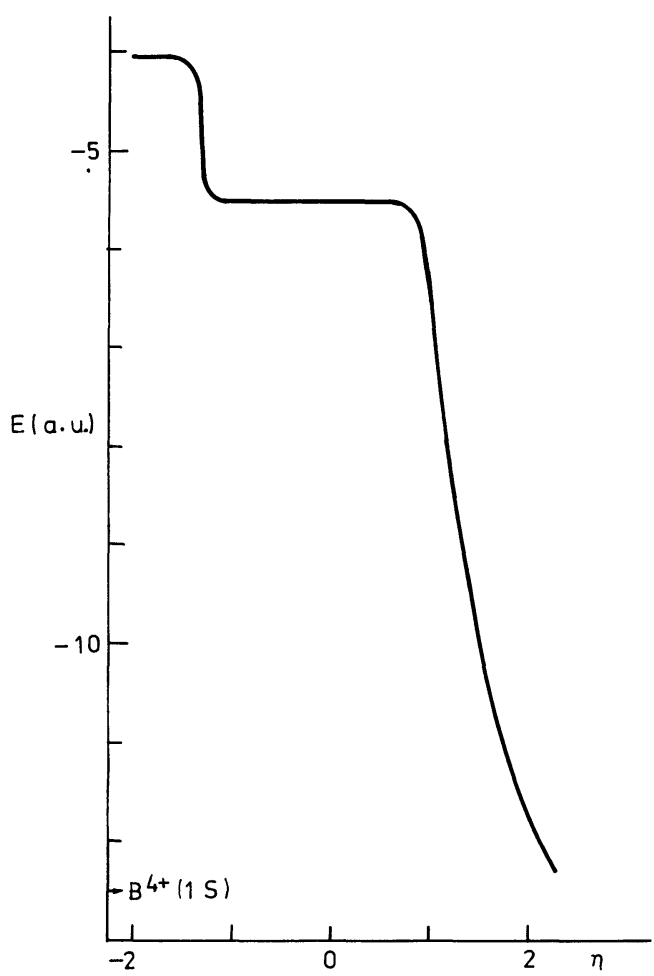

Fig. 2. - Stabilization of one eigenvalue, $E_{12}$, of the Hamiltonian of $\mathrm{B}^{3+}$ in a basis set of ${ }^{1} \mathrm{~S}\left(n \mathrm{~s} n^{\prime} \mathrm{s}\right)$ configurations made up of 1s and 2s STO's (Eq. (3)) with $\alpha_{0_{1 s}}=\alpha_{0_{2 s}}=4$; $\beta_{1 \mathrm{~s}}=\beta_{2 \mathrm{~s}}=0.25 ; i=0, \cdots \pm 2$.

These two examples have allowed us to present the main common characteristics of the method for shape (case 1) and Feshbach (case 2) resonances. If one studies the structure of the wavefunctions (of case 2) in a plateau where the energies are stable, 
one finds that they represent auto-ionizing states in the region of configuration space spanned by the basis set.

\section{Theory.}

To find out how resonances can be selected by the stability property of certain eigenvalues, we first need to discuss some aspects related to the information contained by LIF expansions on the properties of continuum wavefunctions (whether resonant or not). We shall treat the case where the resonant state either dissociates into a particle of coordinate $\mathbf{r}$ and a subsystem, or into two subsystems separated by $\mathbf{r}$. We shall not specify the internal coordinates of the subsystems.

The Hamiltonian, $H$, which represents the system under study can be expressed as

$$
H=\sum_{i} \mid \chi_{i}>E_{i}\left\langle\chi_{i}\left|+\int \mathrm{d} E\right| \chi_{E}>E<\chi_{E}\right|
$$

where the summation corresponds to the discrete spectrum and the integral to the continuum one, and we have chosen standing wave boundary conditions for the latter; and

$$
\begin{gathered}
\left\langle\chi_{i} \mid \chi_{j}\right\rangle=\delta_{i j}, \quad\left\langle\chi_{i} \mid \chi_{E}\right\rangle=0 \\
\left\langle\chi_{E} \mid \chi_{E^{\prime}}\right\rangle=\delta\left(E-E^{\prime}\right) .
\end{gathered}
$$

We also choose $E=0$ as the lower limit of the integral in (5); this can always be done by changing the scale.

In a variational approach one solves

$$
\mathbf{H C}=\mathbf{C} \tilde{\mathbf{E}}
$$

where $\mathbf{H}$ is the Hamiltonian matrix in the LIF (orthonormal) representation. The eigenvectors $c$ then yield the wavefunctions

$$
\Psi_{n}^{\mathrm{s}}(\mathbf{r})=\left\langle\mathbf{r} \mid \Psi_{n}\right\rangle=\sum_{i=1}^{N} c_{i n} \varphi_{i}
$$

For $\widetilde{E}_{n}<0$ the Hylleraas-Undheim-McDonald theorem [6] provides a theoretical basis for the relation between exact $\left(E_{i}\right)$ and approximate $\left(\tilde{E}_{i}\right)$ energies; provided the variational method converges, the former are always lower bounds to the latter. An immediate consequence is that, when any parameter in the basis set is varied, the energies will inevitably go through (at least) one minimum. Thus the stability property of eigenvalues corresponding to bound states is trivially explained. On the other hand, for $\widetilde{E}_{n}>0$, there is no such minimum property of eigenvalues $\left({ }^{2}\right)$; and the stability property of some of these eigenvalues needs a different justification.

$\left({ }^{2}\right)$ Of course we mean for the entire Hamiltonian; if one uses Feshbach [7] projection operators as in Fano's [8] approach, bounds can be obtained for the eigenvalues of the projected Hamiltonian.
To simplify the presentation of the main part of this paper, we first project out the discrete spectrum of $H$, of no interest in the present context; next we introduce a Fine Grain Discretization (FGD) of the continuum spectrum of $H$ : we replace that spectrum by a quasi continuum by enclosing the system in a very large box or using any other suitable device, such that the new eigenfunctions, henceforth called $\bar{\chi}_{E}$, are identical to $\chi_{E}$ inside a (very large) domain $\mathfrak{D}$ of $\mathbf{r}$ space, and are made to vanish quickly (either exponentially or abruptly) outside $\mathfrak{D}$. Notice that since tables and figures always have a finite precision the box can be of macroscopic size, so that there is no practical difference in the spectrum or wavefunctions of the FGD and the exact Hamiltonian; the difference lies in the formal treatment of the continuum which is considerably simplified with the introduction of the FGD. We are left with a new Hamiltonian

$$
\bar{H}=\int_{\varepsilon}\left|\bar{\chi}_{E}\right\rangle E\left\langle\bar{\chi}_{E}\right| \mathrm{d} E
$$

where the integral must be interpreted as a sum over densely packed energy intervals of width $O(\varepsilon)$; that is

$$
\bar{H}=\sum_{i}\left|\bar{\chi}_{E_{i}}\right\rangle E_{i}\left\langle\bar{\chi}_{E_{i}}\right|
$$

When we consider an expression containing $\chi_{E}$ which is a continuous function of $E$; substituting $\chi_{E}$ for $\bar{\chi}_{E}$ in that expression replaces that function by a histogram, which, as mentioned before, would appear in any practical representation as a continuous function. It is then more convenient to use a continuous interpolating function of the histogram. For example, in the particular case of the scalar product, $\left\langle\bar{\chi}_{E_{i}} \mid \bar{\chi}_{E_{j}}\right\rangle$ as a function of $E_{i}$ will be zero for all $E_{i} \neq E_{j}$ and would be very large for $E_{i}=E_{j}$; the interpolating function

$$
\left\langle\bar{\chi}_{E_{i}} \mid \bar{\chi}_{E_{j}}\right\rangle=\delta_{\varepsilon}\left(E_{i}-E_{j}\right)
$$

will then be a member of a $\delta$-family [9] (straightforward extension of a $\delta$ sequence); because in the limit of an infinite box $\bar{\chi}_{E} \rightarrow \chi_{E}, \delta_{\varepsilon}\left(E_{i}-E_{j}\right) \rightarrow \delta\left(E_{i}-E_{j}\right)$.

It is then consistent to choose the area below the interpolating function such that

$$
\int \mathrm{d} E^{\prime} \delta_{\varepsilon}\left(E-E^{\prime}\right)=1
$$

and one also has by construction

$$
\delta_{\varepsilon}\left(E-E^{\prime}\right)=0 \text { when } \quad\left|E-E^{\prime}\right|>\varepsilon
$$

so that

$$
\left\langle\bar{\chi}_{E_{i}} \mid \bar{\chi}_{E_{i}}\right\rangle=\delta_{\varepsilon}(0)=O\left(\varepsilon^{-1}\right) .
$$

Let us now turn to the discretization of the spectrum of the Hamiltonian $H$ which is provided by a variational treatment. Usually this discretization differs strongly from the FGD we have just discussed. 
This is because the LIF basis used spans a region D of $\mathbf{r}$ space which is of molecular dimensions and very small compared to, and included in, the large domain $\mathcal{D}$ (where $\chi_{E}$ and $\bar{\chi}_{E}$ are identical). The energy levels $E_{n}$ obtained in actual variational calculations are quite separate so that these calculations are equivalent to a coarse grain discretization (CGD) as far as the continuum is concerned. In other words, the domain $\mathfrak{D}$ is chosen so large that the results of actual variational treatment for $H$ and $\bar{H}$ will yield the same results.

An advantage of having introduced FGD is that $\bar{\chi}_{E}$ can be, in principle, reproduced as well as desired by increasing indefinitely the basis set of LIF. Of course, we are not actually going to the limit of a complete basis set but we can consider this limit formally. For example, suppose we want to know some properties of an expression of $\bar{\chi}_{E}$ as a (interpolating) function of $E$. For a reasonably good basis set this behaviour can be sometimes gleaned if it can be formally known in the case of a complete basis set.

The energy levels $E_{n}$ of equation (7) are related to the characteristics of the eigenfunctions of $\bar{H}$ and of the basis set; one has

$$
\tilde{E}_{n}=\left\langle\Psi_{n}|H| \Psi_{n}\right\rangle=\int_{\varepsilon} \Psi_{n}^{\mathrm{H}}(E)^{2} E \mathrm{~d} E
$$

where $\Psi_{n}^{\mathrm{H}}(E)$ is the Heisenberg representation of $\left|\Psi_{n}\right\rangle$ defined by

$$
\Psi_{n}^{\mathrm{H}}(E)=\left\langle\bar{\chi}_{E} \mid \Psi_{n}\right\rangle
$$

and the eigenvalues of $\bar{H}$ are given as the average value of $E$ with respect to a weight function $\Psi_{n}^{\mathrm{H}}(E)^{2}$.

To know the behaviour of $\Psi_{n}^{\mathrm{H}}(E)$ as a function of $E$ for the usual type of basis set we can use the procedure mentioned above, which is to go formally to the limit $N \rightarrow \infty$. Notice, therefore, that the following equations $(17,18,19$ and 20) are of formal value and only used to learn about the properties of $\Psi_{n}^{\mathrm{H}}(E)$. When the basis set becomes complete, the spectrum of $\tilde{E}_{n}$ tends to the quasi continuum spectrum of $\bar{H}$ and

$$
\Psi_{n}^{\mathrm{s}}(\mathbf{r}) \rightarrow \delta_{\varepsilon}^{-1 / 2}(0) \bar{\chi}_{\widetilde{E}_{n}}(\mathbf{r})
$$

as can be shown from

$$
\delta_{n m}=\left\langle\Psi_{n} \mid \Psi_{m}\right\rangle \rightarrow \delta_{\varepsilon}^{-1}(0)\left\langle\bar{\chi}_{\tilde{E}_{n}} \mid \bar{\chi}_{\tilde{E}_{m}}\right\rangle .
$$

Using equation (11), equation (18) then becomes :

$$
\rightarrow \delta_{\varepsilon}^{-1}(0) \delta_{\varepsilon}\left(\tilde{E}_{n}-\widetilde{E}_{m}\right)=\delta_{n m}
$$

since $\left|\widetilde{E}_{n}-\widetilde{E}_{m}\right|>\varepsilon$. Hence the normalization constant in equation (17) is correct, and corresponds to the intuitively obvious fact that the amplitude of the ( $S$-normalized) wavefunction $\Psi_{n}^{\mathrm{s}}(\mathbf{r})$ must become progressively smaller as the basis set is enlarged.
From equations (11), (16) and (17) one finds

$$
\Psi_{n}^{\mathrm{H}}\left(\tilde{E}_{i}\right)^{2} \rightarrow \delta_{\varepsilon}^{-1}(0)\left\langle\bar{\chi}_{\tilde{E}_{i}} \mid \bar{\chi}_{\tilde{E}_{n}}\right\rangle^{2}=\delta_{i n} \delta_{\varepsilon}(0)
$$

that is, in the limit $N \rightarrow \infty, \Psi_{n}^{\mathrm{H}}(E)^{2}$ becomes completely concentrated about $\widetilde{E}_{n}$. This indicates that for reasonably large basis sets one can expect that $\Psi_{n}^{\mathrm{s}}(\mathbf{r})$ will have a maximum overlap with the continuum wavefunction $\bar{\chi}_{E}(\mathbf{r})$. In practice, the corresponding Heisenberg transform $\Psi_{n}^{\mathrm{H}}(E)$ (which is an interpolating function of the energy) is non-negligible in a relatively small interval of width $\Delta_{n}$ about $\widetilde{E}_{n}$, which nevertheless will contain a large number of eigenvalues of $\bar{H}$. The spectrum obtained in solving equation (7) then depends, through equations (15) and (16), on the properties of both $\Psi_{n}^{\mathrm{s}}(\mathbf{r})$ as a function of the basis set and on $\bar{\chi}_{E}(\mathbf{r})$.

When using the stabilization method - that is, some parameters in the finite basis set (Eq. (4)) are varied - unless $\bar{\chi}_{E}(\mathbf{r})$ has some particular property, this variation will result in a concomitant variation of the region $\Delta_{n}$ where $\Psi_{n}^{\mathrm{H}}(E)$ is non-negligible. As a consequence of equation (15), $\widetilde{E}_{n}$, around which $\Delta_{n}$ is centred, will also vary with the basis set, yielding what is called an unstable eigenvalue of equation (7).

Now assume there is a resonance for $E=\xi$. The picture of a resonance as a discrete state embedded in, and coupled to, a continuum was introduced by Feshbach [7, 10]. Another definition of resonance makes use of the analytic continuation of the (scattering) $S$-matrix into the complex energy or momentum plane [11]; an $S$-matrix pole is located at the energy of either a bound state, a virtual state or a resonance. When using the stabilization method one is normally interested in resonances which are very similar to bound states : we can characterize such resonances by the fact that, for a value of $r$ in the domain $\mathrm{D}$ which is of molecular dimensions (hence $\mathrm{D} \ll \mathcal{D}$ ), the resonant wavefunction has an amplitude which is maximal relative to that of any wavefunction corresponding to neighbouring energies.

We can then write (see Appendix)

$$
\bar{\chi}_{E}(\mathbf{r})=A(E) \hat{\chi}_{E}(\mathbf{r})
$$

where $A(E)$ presents a sharp peak for the resonance energy, $E=\xi$. For $\mathbf{r} \in \mathrm{D}$ (and only there) we have thus factorized the maximum relative amplitude of $\bar{\chi}_{E}(r)$ as a function of $E$.

For $E \cong \xi$ one has :

$$
\Psi_{n}^{\mathrm{H}}(E)=A(E) \int \Psi_{n}^{\mathrm{s}}(\mathbf{r}) \hat{\chi}_{E}(\mathbf{r}) \mathrm{d} \mathbf{r}=A(E) \hat{\Psi}_{n}^{\mathrm{H}}(E)
$$

where

$$
\hat{\Psi}_{n}^{\mathrm{H}}(E)=\left\langle\hat{\chi}_{E} \mid \Psi_{n}\right\rangle
$$

Because of the properties of the basis set, which spans preferentially the region $D$ of $\mathbf{r}$ space, the dominant contribution to the integral in equation (22) 
corresponds to D. Since, for $\mathbf{r} \in \mathrm{D}$, we have factored out the energy peaking structure peculiar to the resonance in $\hat{\chi}_{E}(\mathbf{r})$, one can expect that, similarly to $\Psi_{n}^{\mathrm{H}}(E)$ for the non-resonant case, $\hat{\Psi}_{n}^{\mathrm{H}}(E)$ will be negligible outside a (relatively small) region $\hat{\Delta}_{n}$, which again, will be unstable upon variation of the basis set. However, as

$$
\widetilde{E}_{n}=\int A(E)^{2} \hat{\Psi}_{n}^{\mathrm{H}}(E)^{2} E \mathrm{~d} E
$$

as long as the maximum of $A^{2}(E)$ falls within the region $\hat{\Delta}_{n}, \widetilde{E}_{n}$ will closely correspond to the position of the maximum value. In other words, the eigenvalue is stable.

Since the maximum of $A(E)$ corresponds to the position of the resonance, we reach our explanation of how the stabilization of some eigenvalues is achieved. We have schematized our reasoning in figure 3. One varies the basis set, and this brings about a change in the position of $\hat{\Delta}_{n}$; as long as $\hat{\Delta}_{n}$ does not enclose a resonance energy, the method yields an unstable eigenvalue. On the other hand, for all basis sets such that $\hat{\Delta}_{n}$ encloses a resonance energy one obtains an eigenvalue which closely approximates that energy.
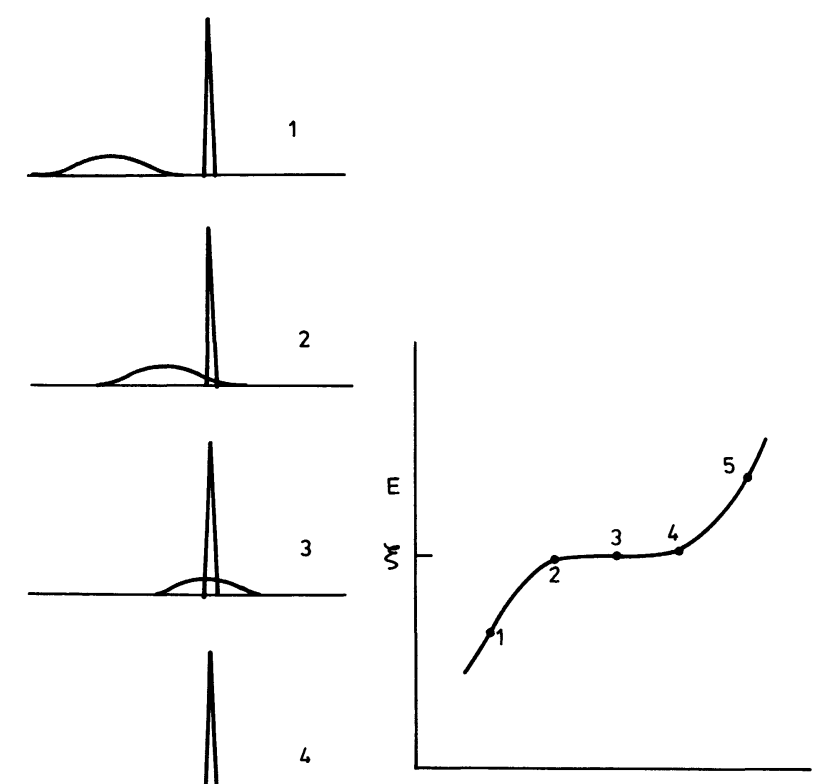

Variation in basis set

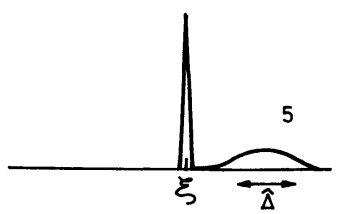

Fig. 3. - Explanation of the stability of an eigenvalue when a resonance energy falls in the range $\hat{\Delta}_{n}$, where the Heisenberg transform is non-negligible. The sharp peak corresponds to the maximum of $A^{2}(E)$ (See Eq. (24)).
It will be noticed that our analysis does not constitute a proof in the strict sense, because our expectation that $\hat{\Psi}_{n}^{\mathrm{H}}$ is negligible outside a relatively wide (unstable) interval $\hat{\Delta}_{n}$ cannot in general be proved. When that expectation is not fulfilled, stabilization is highly unlikely : we present an illustration of this point in section 5 .

Finally, the core of our explanation of why the stabilization method works is seen to rely on two facts : the energy peaking structure of the wavefunction (Eq. (21)) in the domain D and the preferential description of this domain by the basis set used. Turning back to the examples of section 2 we see that the domain $\mathrm{D}$ corresponds to the well in case 1 , and in case 2 (Feshbach resonance) it corresponds to a sphere of radius $\sim 0.5$ a.u. about the nucleus. In both cases the STO's used are centred at $r=0$ so that the basis spans preferentially the region $D$. We think that our heuristic explanation provides the justification for the intuitive use of variation of the basis sets to stabilize resonances.

\section{Illustration.}

We first consider the trivial case of $H=-1 / 2 \nabla^{2}$, which has a purely continuum spectrum and presents no resonances. We have solved equation (7) in the basis set of even tempered STO's used in our first example, with three different basis sets corresponding to three different values of $\eta$ (see Eq. (4)). We present in figure 4 the values of $k \Psi_{n}^{\mathrm{H}}(E)^{2}$ as functions of $k=\sqrt{2 E}$. We have chosen $k$ as a variable because we find it better for illustrative purposes; notice the equivalence

$$
\tilde{E}_{n}=\int \Psi_{n}^{\mathrm{H}}(E)^{2} E \mathrm{~d} E=\int k \Psi_{n}^{\mathrm{H}}(E)^{2} E \mathrm{~d} k
$$

In all three cases $k \Psi_{5}^{\mathrm{H}}(E)^{2}$ presents a relatively broad maximum centred very near $k_{5}=\sqrt{2 \widetilde{E}_{5}}$; the function $\delta_{\varepsilon}\left(\widetilde{E}_{5}-E\right)$ of equation (20) is replaced by a rather broad peak of width $\Delta_{5} \simeq k_{6}-k_{4}$. All eigenvalues are unstable (they vary widely with $\eta$ ); in fact, in this case $\widetilde{E}_{n}=$ Const. $\eta^{2}$ because of the homogeneous property of $\nabla^{2}$.

Let us now add to the model of the previous example a potential able to support resonances. To calculate $\Psi_{n}^{\mathrm{H}}(E)^{2}$ we need to know the exact solutions $\chi_{E}$. For this reason we choose

$$
H=-\frac{1}{2} \nabla^{2}+\Omega \delta(r-1)
$$

whose solutions can be expressed in analytic form [12]. In the notation of equation (21) :

$$
\begin{array}{r}
A(E)=\left(1+\frac{2 \Omega}{k} \sin 2 k+\frac{4 \Omega^{2}}{k^{2}} \sin k^{2}\right)^{-1 / 2}= \\
=\sin (k+\delta) / \sin k
\end{array}
$$


and

$$
\begin{array}{rlrl}
\hat{\chi}_{E}(\mathbf{r}) & =\frac{1}{\pi r(2 k)^{1 / 2}} \sin k r & & 0<r<1 \\
& =\frac{1}{A(E) \pi r(2 k)^{1 / 2}} \sin (k r+\delta) & r>1 .
\end{array}
$$

Because of the simple form of the Hamiltonian (Eq. (25)) a more accurate form of $A(E)$ is given than that in the appendix. We are thus able to extract exactly the energy peaking structure of $\chi_{E}$ (or $\bar{\chi}_{E}$ ) for $r \in \mathrm{D}=$ $[0,1]$ with the single analytic form (26). As explained before, the presence of $A(E)$ in the denominator of $\hat{\chi}_{E}(r)$ for $r>1$ is of no importance because the basis set describes preferentially the domain D (Eq. (22)). We take $\Omega=10$ which is large enough for all definitions of resonance positions to yield practically the same results. For example, in terms of the logarithmic derivative, we have, for $E=\xi$

$$
\kappa \operatorname{cotg} \kappa+2 \Omega=0
$$

where

$$
\kappa=\sqrt{2 \xi}
$$

and we obtain an infinity of resonance energies

$$
\begin{aligned}
\xi_{n} & =\frac{n^{2} \pi^{2}}{2}\left(1-\frac{1}{\Omega}+\frac{3}{4} \frac{1}{\Omega^{2}}+\cdots\right) \\
n & =1,2, \ldots
\end{aligned}
$$

In figure 5a we present a plot of $A(E)$, which has very sharp peaks at the positions $\xi_{n}$. When $H$ (or $\left.\bar{H}\right)$ in equation (25) is diagonalized in the same even tempered basis set of our first example in section 4, we obtain eigenvalues which present a plateau region near the position of the resonance energies (Fig. 5b), quite similar to those of figures 1 and 2 .

In figure 6 we also present a plot of the exact and variational wavefunctions corresponding to the first resonance energy, $\xi_{1}$. The exact wavefunction, which

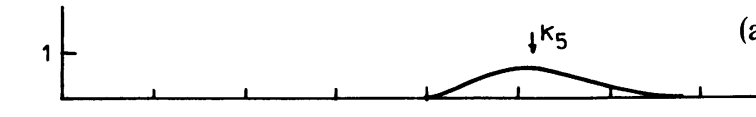

(a)

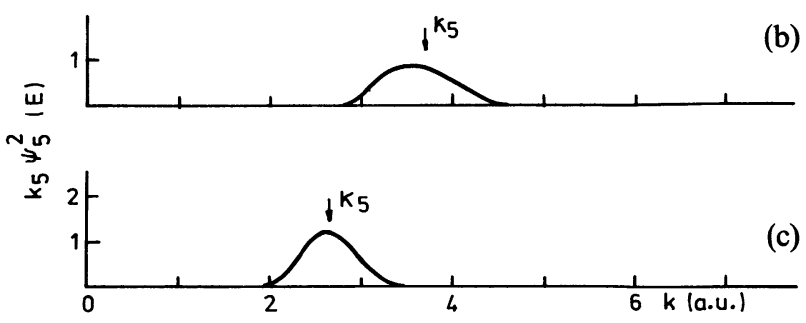

Fig. 4. - Values of $k \Psi_{5}^{2}(E)$ as functions of $k=\sqrt{2 E}$, where $\Psi_{5}(E)$ is the Heisenberg representation of the fifth eigensolution of equation (11) for $H=-1 / 2 \nabla^{2}$. Arrows indicate the position of the eigenvalues. Basis set of $1 \mathrm{~s}$ STO's with $\alpha_{0}=14.06 ; i=0, \cdots \pm 9 ; \beta=0.8$; (a) $\eta_{1}=-1.6$, (b) $\eta_{2}=0$, (c) $\eta_{3}=1.4$.

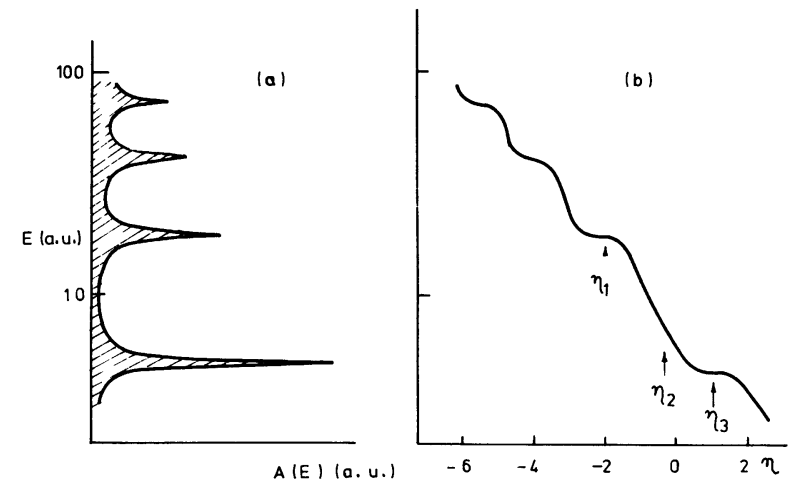

Fig. 5. - Plot of the maxima of $A(E)$ vs. $E$ (a), and stabilization of the fifth eigenvalue of equation (21) (b) at the resonance positions indicated by the maxima of $A(E)$. Basis sets as in figure 4. The values of the scaling parameter are indicated in stable $\left(\eta_{1}\right.$ and $\left.\eta_{3}\right)$ and unstable $\left(\eta_{2}\right)$ regions.

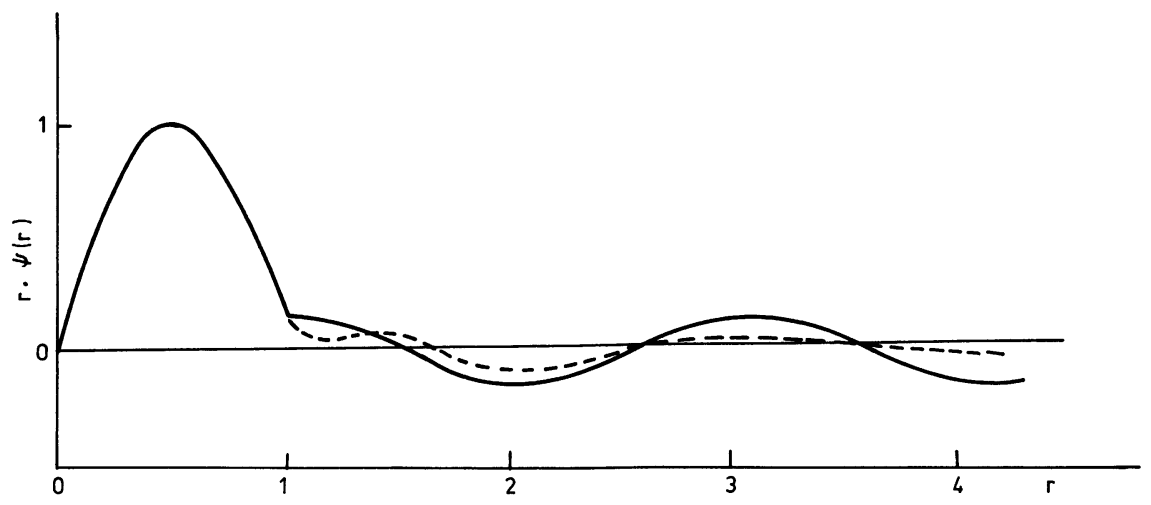

Fig. 6. - Exact (- - ) and variational (----) wavefunctions corresponding to the first resonance energy of the Hamiltonian in equation (25) with $\Omega=10$ (Basis set $\eta_{3}$ of Fig. 5). Normalizing both wavefunctions to have a maximum amplitude of 1 , they are indistinguishable in the inner region of the $\delta$-shell potential. 
has a relative maximum amplitude in the region $r<1$, is very well reproduced in that region by the basis set; and outside it the reproduction becomes poorer as $r$ increases. More « excited " resonances are similarly reproduced; they are not presented here for conciseness.

We illustrate the behaviour of just one eigenvalue both in a plateau and in the unstable regions, taking three values of $\eta$ (hence three basis sets) called $\eta_{1}, \eta_{2}$ and $\eta_{3}$ in figure $5 \mathrm{~b}$, and we plot in figure 7 , just as in figure $4, k \Psi_{n}^{2}(E)$ as a function of $k=\sqrt{2 E}$.

Using the notation of the previous section, figure 7a corresponds to the case when $\xi_{2} \in \hat{\Delta}_{5}$, and the peak in $A^{2}(E)$ completely dominates the integrand (in Eq. (24)) then yielding $\widetilde{E}_{5} \simeq \xi_{2} ;$ this occurs in the whole plateau region as schematized in figure 3 . When the basis set $\left(\eta_{2}\right)$ is such that neither $\xi_{1}, \xi_{2} \in \hat{\Delta}_{5}$, (Fig. 7b) the eigenvalue is unstable with respect to variations of $\eta$. Finally (Fig. 7c) when $\eta=\eta_{3}$ we have $\xi_{1} \in \hat{\Delta}_{5}$. Just as before the eigenvalue is stable but it now yields $\widetilde{E}_{5} \cong \xi_{1}$.

\section{Additional conclusions.}

From our explanation of the basis of the stabilization method one can draw the following conclusions.

Firstly, from a practical point of view, when the stabilization method is used to detect and approximate resonances, employing the kind of $L^{2}$ integrable functions common in the calculation of bound states, one should not just enlarge the basis set, but vary it (e.g. vary $\eta$ in Eq. (4) for an even tempered basis) to detect resonances; and increase the denseness of the basis (decrease $\beta$ ) in order to improve the representation.

Secondly, let us suppose that we use a very large basis set such that we practically obtain the limit CGD $\rightarrow$ FGD considered in section 3. We are then able to reproduce with very good precision the wavefunctions of the continuum spectrum of $H$. Surprisingly, as our reasoning shows, the stabilization method considered here will then not work. This is because, in this case, the Heisenberg transform $\hat{\Psi}_{n}(E)^{2}$ has an almost $\delta$-function shape (approaching Eq. (20)) so that the energy band, $\hat{\Delta}_{n}$, is extremely narrow and so will be the plateau region where the corresponding eigenvalue is stable; then the eigenvalue would appear to be unstable (Fig. 8). Stabilization then requires not an extremely good representation of continuum wavefunctions but rather that the basis set span preferentially the region of $\mathbf{r}$ space where the resonant wavefunction has maximum amplitude, and the complementary region be described grossly. Then, in practice, the basis set must be carefully chosen : making it nearly complete is not an alternative and may even become a liability. Also, it can be noticed that there are practical limitations on the maximum density that can be achieved, because as $\beta \rightarrow 1$ there. appear quasi-linear dependencies in the basis set.
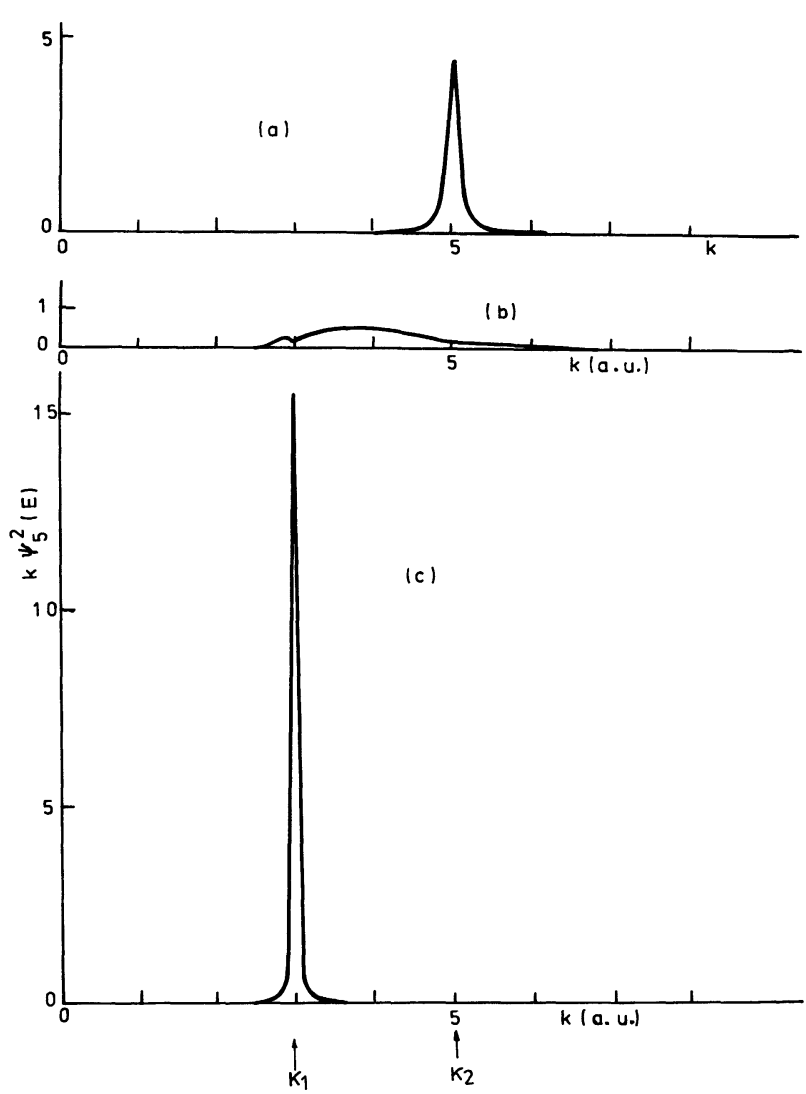

Fig. 7. - Values for $k \Psi_{5}^{2}(E)$ as functions of $k=\sqrt{2 E}$ where $\Psi_{5}(E)$ is the Heisenberg representation of the fifth eigenstate of the Hamiltonian (Eq. 21) for the basis sets indicated by (a) $\eta_{1}$, (b) $\eta_{2}$ and (c) $\eta_{3}$ of figure 4. The shape of $k \Psi_{5}^{2}(E)$ remains the same as in the figure as long as the eigenvalue is stabilized (in a plateau region). These results correspond to the product of the two curves in the qualitative diagram of figure 3.

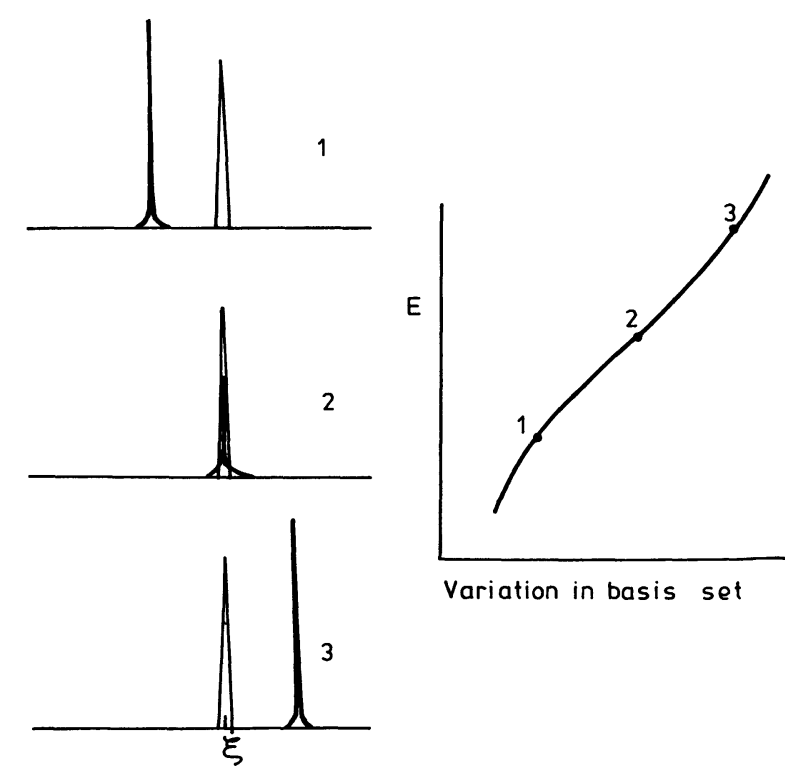

Fig. 8. - Explanation of why improving the description of a resonance by increasing the denseness of the basis set can have the negative consequence of making the plateau region so small that the resonance is not detected, (compare with Fig. 3). 
Thirdly, the point discussed in the previous paragraph poses the practical question of how well a resonance can be reproduced by the stabilization method. Suppose one has stabilized a given resonance to a good approximation, but one would still like to improve the precision of this representation. The obvious way is to enlarge the basis set, which yields a better representation of all continuum wavefunctions, but a limit is reached where detection of resonances becomes extremely difficult because the plateau regions are too small. When one desires to indefinitely optimize the approximation to a resonance wavefunction reached in a variational treatment, one can then resort to a variance-type method which has been shown to converge (e.g. in the specific case of the Stark effect [13]).

Finally, when it so happens that the Heisenberg transform spans more than one resonance the integrand of equation (24) presents more than one sharp peak, and therefore, the eigenvalue falls between the corresponding values and does not yield that of any one resonance. A particular instance where this situation is likely to occur is the case of very close (or overlapping) resonances, where the stabilization method will have difficulty in distinguishing between them. An obvious consequence is that sharp isolated resonances are the easiest ones to stabilize.

\section{Acknowledgments.}

Calculations have been carried out at the 1110 UNIVAC computer at the J.E.N. (Madrid).

\section{Appendix.}

In this appendix we shall justify the factorization (Eq. (21)) of $\bar{\chi}_{E}(\mathbf{r})$ into an $\mathbf{r}$ dependent part $\hat{\chi}_{E}(\mathbf{r})$ and a factor $A(E)$ which presents a sharp peak about the resonance energy $E=\xi$. Many forms for $A(E)$ are possible; as an illustration, we present our reasoning for a simple Gaussian form.

In the text we have characterized the kind of resonance to be stabilized by the fact that the corresponding wavefunction, $\bar{\chi}_{E}(\mathbf{r})$ has, for a value of $\mathbf{r}$ in a domain $\mathrm{D}$ of molecular dimensions, an amplitude which is maximum relative to that of wavefunctions corresponding to neighbouring energies. Then one can write

$$
\ln \bar{\chi}_{E}(\mathbf{r})=G_{0}(\mathbf{r})-G_{1}(\mathbf{r})(E-\xi)^{2}+\cdots
$$

so that

$$
\bar{\chi}_{E}(\mathbf{r})=\exp \left(-G_{1}(\mathbf{r})(E-\xi)\right)^{2} \cdot \Xi_{E}(\mathbf{r})
$$

for $E$ in the neighbourhood of $\xi$. When the domain D is not very extensive one can choose an intermediate value $r_{0} \in D$ such that

$$
G_{1}(\mathbf{r})=G_{1}\left(\mathbf{r}_{0}\right)+G_{1}^{\prime}\left(\mathbf{r}_{0}\right)\left(\mathbf{r}-\mathbf{r}_{0}\right)+\cdots
$$

converges quickly in $\mathrm{D}$. One can then write

$$
\begin{aligned}
\bar{\chi}_{E}(\mathbf{r}) & =\exp \left(-G_{1}\left(\mathbf{r}_{0}\right)(E-\xi)^{2}\right) \hat{\chi}_{E}(\mathbf{r}) \\
& =A(E) \hat{\chi}_{E}(\mathbf{r})
\end{aligned}
$$

which defines both $A(E)$ and $\hat{\chi}_{E}(\mathbf{r})$ and yields equation (21) of the text.

In the special case of the domain $D$ being such that equation (A.3) does not converge quickly, then equation (A.2) must be used instead of equation (21) in the text. Equation (22) still holds, however, because one can divide the domain $\mathrm{D}$ into (a few) subdomains $D_{j}$ where the Taylor series of $G_{1}(r)$ converges quickly, yielding

$$
\bar{\chi}_{E}\left(\mathbf{r} \in \mathrm{D}_{j}\right)=\exp \left(G_{1}\left(\mathbf{r}_{0 j}\right)(E-\xi)^{2}\right) \hat{\chi}_{E}\left(\mathbf{r} \in \mathrm{D}_{j}\right) .
$$

The Heisenberg transform $\Psi_{n}^{\mathrm{H}}(E)$ can then be written as a sum of contributions from each of the domains :

$$
\begin{aligned}
\Psi_{n}^{\mathrm{H}}(E)=\sum_{j} \exp \left(-G_{1}\left(\mathbf{r}_{0 j}\right)(E-\xi)^{2}\right) \times \\
\times \int_{\mathrm{D}_{j}} \Psi_{n}^{\mathrm{s}}(\mathbf{r}) \hat{\chi}_{E}(\mathbf{r}) \mathrm{d} \mathbf{r} .
\end{aligned}
$$

The reasoning involved in equations (A.1) and (A.2) can now be repeated leading again to the last member in equation (22) in the text. For simplicity we present the case where (A.4) is a good approximation since this entails, as we have just shown, no loss of generality. 


\section{References}

[1] O'Malley, T. F., Adv. Atom. Molec. Phys. 7 (1971) 223.

[2] Macias, A. and Riera, A., Phys. Reports 81 (1982) 299.

[3] Taylor, H. S., Adv. Chem. Phys. 18 (1970) 91 ;

Hazi, A. U. and Taylor, H. S., Phys. Rev. A 11109 (1970);

Fels, M. F. and Hazi, A. U., Phys. Rev. A 4 (1971) 662, Ibid., Phys. Rev. A 5 (1972) 1236.

[4] RuEdenberg, K., RAfFenetti, R. C. and BARdo, R. D., Energy, structure and reactivity Proc. 1972 Boulder Conf. on Theoretical Chemistry (Wiley, N. Y.) 1975.

[5] Mactas, A., Mendizabal, R., Pelayo, F., Riera, A. and YÁÑEZ, M., J. Mol. Struct. Theochem. 107 (1984) 245.

[6] Hylleraas, E. A. and Undheim, B., Z. Phys. 65 (1930) 759 ;
McDonald, J. K. L., Phys. Rev. 43 (1933) 830.

[7] Feshbach, H., Ann. Phys. (N. Y.) 19 (1962) 287.

[8] FANO, U., Phys. Rev. 124 (1961) 1866.

[9] Cohen-Tannoudj, C., DiU, B. and Lalö̈, F., Mécanique quantique I (Hermann Paris) 1980;

STAKGOLD, I., Boundary value problems of Mathematical Physics, Vol. I (McMillan, London) 1967.

[10] Feshbach, H., Ann. Phys. N. Y. 5 (1958) 357.

[11] JoACHaIN, C. J., Quantum collision theory (North Holland, Amsterdam) 1975;

NEwTON, R. G., Scattering theory of waves and particles (McGraw-Hill, N.Y.) 1966.

[12] GotTFried, K., Quantum mechanics, Vol. 1 (Benjamin, N.Y.) 1966 ;

Flugge, S., Practical Quantum Mechanics (Springer, N.Y.) 1974.

[13] Macías, A. and Riera, A., J. Phys. B 13 (1980) L449. 